\title{
Agradecimento aos revisores
}

DOI: https://dx.doi.org/10.7322/abcshs.v43i1.1161

A ABCS Health Sciences agradece a valiosa colaboração de seus revisores científicos em 2017:

- Adriana Teresa Silva - UNIVÁS - Pouso Alegre/MG

- Alberto Manuel Quintana - UFSM - Santa Maria/RS

- Alexander Garcia Perker - UFFS - Chapecó/SC

- Alexandre Almeida - HSCS - Caxias do Sul/RS

- Altiere Araujo Carvalho - Unimarco - São Paulo/SP

- Ana Carolina Fonseca Lindoso Melo - UFC - Fortaleza/CE

- Ana Carolina Sartorato Beleza - UFSCar -São Carlos/SP

- Ana Eliza Rios de Araújo Mathias - UPE - Petrolina/PE

- Andy Petroianu - UFMG - Belo Horizonte/MG

- Angela Amorim de Araújo - UFPB - João Pessoa/PB

- Antonio Pedro Ricomini Filho - UNICAMP - Campinas/SP

- Apoena de Aguiar Ribeiro - UFF - Nova Friburgo/RJ

- Bianca Schmid - IBGE - São Paulo/SP

- Carla Elaine Laurienzo da Cunha Andrade - HCB - Barretos/SP

- Carmen Silvia da Silva Martini - UFAM - Manaus/AM

- Carolina Terra de Moraes Luizaga - FOSP - São Paulo/SP

- Cássia Regina Vancini-Campanharo - UNIFESP - São Paulo/SP

- Cassiana Mendes Bertoncello Fontes - UNESP - Botucatu/SP

- Cesar Junior Aparecido de Carvalho - IFPR - Londrina/PR

- Cintia Bezerra Almeida Costa - UFPB - João Pessoa/PB

- Cláudia da Silva Funchal - IPA - Porto Alegre/RS

- Cláudia de Castro Selestrin - FMABC - Santo André/SP

- Claudia Lins Cardoso - UFMG - Belo Horizonte/MG

- Claudia Salvini Barbosa Martins da Fonseca - FMP Petrópolis/RJ

- Claudio Luders - USP - São Paulo/SP

- Cleci de Fátima Enderle - FURG - Rio Grande/RS

- Cristiane Funghetto Fuzinatto - UFFS - Chapecó/SC

- Cristiane Kopacek - HMIPV - Porto Alegre/RS

- Cristina Maria Nunes Cabral - UNICID - São Paulo/SP

- Daisy Maria Xavier de Abreu - UFMG - Belo Horizonte/MG

- Daniel Cury Ogata - UNIVALI - Itajaí/SC

- Daniele de Almeida Soares Marangoni - UFMS - Campo Grande/MS

- Darlene Mara dos Santos Tavares - UFTM - Uberaba/MG

- Débora Driemeyer Wilbert - FMU - São Paulo/SP

- Deivis Elton Schlickmann Frainer - IFC - Blumenau/SC

- Denise Maria Christofolini - FMABC - Santo André/SP

- Elaine Gagete Miranda da Silva - UNESP - Botucatu/SP

- Elcio Luiz Bonamigo - UNOESC - Joaçaba/SC
- Elder dos Santos Cavalcante - UNIFESP - São Paulo/SP

- Elizabete Regina Araújo de Oliveira - UFES - Vitória/ES

- Elizabeth Ferreira Rangel - FIOCRUZ - Rio de Janeiro/RJ

- Eloá Ferreira Yamada - Unipampa - Uruguaiana-RS

- Eveline Franco da Silva - FSG - Caxias do Sul/RS

- Ezequiel Aparecido dos Santos - UNESP - Botucatu/SP

- Fabiana de Andrade Bringel - ITPAC - Araguaína/GO

- Fábio Chigres Kuschnir - UERJ - Rio de Janeiro/RJ

- Fátima Antonia Aparecida Zanin - Unicastelo - São Paulo/SP

- Flavia Aparecida Dias - UFTM - Uberaba/MG

- Flávia Martins Gervásio - UEG - Anápolis/GO

- Franciéle Marabotti Costa Leite - UFES - Vitória/ES

- Francisca Maria de Almeida - UFSC - Florianópolis/SC

- Francisco José Machado Viana - CNP - Belo Horizonte/MG

- Geraldo Alberto Sebben - PUC - Curitiba/PR

- Geysa Aguiar Romeu - Unifor - Fortaleza/CE

- Guilherme da Silva Gasparotto - IFPR - Pinhais/PR

- Gustavo Silveira Borges - UESC - Criciúma/SC

- Inês Gimenes Rodrigues - UEL - Londrina/PR

- Isabela Saraiva de Queiroz - PUC - Belo Horizonte/MG

- Isabella Dib Ferreira Gremião - FIOCRUZ - Rio de Janeiro/RJ

- Isabelle Campos de Azevedo - UFRN - Natal/RN

- Jamila Alessandra Perini Machado - UEZO - Rio de Janeiro/RJ

- Janaina Soares - USP - São Paulo/SP

- Janete Vettorazzi - UFRGS - Porto Alegre/RS

- Janeth de Oliveira Silva Naves - UnB - Brasília/DF

- Jéssica Nunes Silva - FIOCRUZ - Rio de Janeiro/RJ

- José Angelo Barela - UNICSUL - São Paulo/SP

- Júlia Guimarães Reis - USP - Ribeirão Preto/SP

- Julio Cesar Gali - PUC - São Paulo/SP

- Laura Alves Cabral - UFJF - Governador Valadares/MG

- Licia Santos Santana - USP - Ribeirão Preto/SP

- Ligia Christina Borsato Guimarães Nunes - UNICAMP Campinas/SP

- Ligia Petrolini de Oliveira - UFABC - Santo André/SP

- Liliane Gontan Timm Della Mea - UFSM - Santa Maria/RS

- Lisiane Schilling Poeta - UFSC - Florianopolis/SC

- Livio Cesar Cunha Nunes - UFPI - Teresina/PI

- Luciana Zaranza Monteiro - UDF - Brasília/DF

- Luciane Cristina Jóia - FASB - Barreiras/BA 
- Luis Paulo Gomes Mascarenhas - UNICENTRO - Riozinho/PR

- Luísa Pedrosa de Albuquerque Sousa - UFRJ - Rio de Janeiro/RJ

- Luiz Guilherme Martins Castro - HIAE - São Paulo/SP

- Maíra Ainhoren Meimes - UFRGS - Porto Alegre/RS

- Márcia Maria Oliveira Lima - UFVJM - Diamantina/MG

- Márcio Oliveira - UnB - Brasília/DF

- Marco Aurélio Vinhosa Bastos Jr - UFMS - Campo Grande/MS

- Maria Andréa Fernandes - UFPB - João Pessoa/PB

- Maria Auxiliadora Nogueira Saad - UFF - Niterói/RJ

- Maria Cristina Martins de Almeida Macedo - IBCC São Paulo/SP

- Maria Cristina Pinto de Jesus - UFJF - Juiz de Fora/MG

- Maria das Graças Gazel de Souza - INCA - Rio de Janeiro/RJ

- Maria do Rosário Gondim Peixoto - UFG - Goiânia/GO

- Maria Eliane Moreira Freire - UFPB - João Pessoa/PB

- Maria Goreti da Silva Cruz - UNIFESP - São Paulo/SP

- Marilu Fiegenbaum - UFCSPA - Porto Alegre/SP

- Marina Raijche Mattozo Rover - UFSC - Florianópolis/SC

- Mauro Célio de Almeida Marzochi - FIOCRUZ - Rio de Janeiro/RJ

- Milton Marchioli - FAMEMA - Marília/SP

- Nalú Pereira da Costa Kerber - FURG - Rio Grande/RS

- Ney Pereira Carneiro dos Santos - UFPA - Belém/PA

- Patrícia Fernanda Roesler Bertolini- PUC - Campinas/SP

- Patrícia Zaidan de Barros - UERJ - Rio de Janeiro/RJ

- Paulo Ricardo Criado - USP - São Paulo/SP
- Pedro Cláudio Gonsales de Castro - USP - São Paulo/SP

- Pedro Henrique Berbert de Carvalho - UFJF - Juiz de Fora/MG

- Pedro Henrique Scheidt Figueiredo - UFVJM - Diamantina/MG

- Rafael Silva Marconato - UNICAMP - Campinas/SP

- Raquel de Mendonça Nepomuceno - UERJ - Rio de Janeiro/RJ

- Regiani Guarnieri - FMABC - Santo André/SP

- Regina Coeli Marques de Carvalho - UFC - Fortaleza/CE

- Reginaldo Peçanha Brazil - FIOCRUZ - Rio de Janeiro/RJ

- Rejane Barbosa de Oliveira - UTFPR - Santa Helena/PR

- Roberta Kaliny de Souza Costa - UERN - Caicó/RN

- Roberto Jerônimo dos Santos Silva - UFS - São Cristóvão/SE

- Rodolfo de Paula Vieira - UNINOVE - São Paulo/SP

- Rosa Helena Silva Souza - UFPR - Curitiba/PR

- Rosane Azevedo Neves da Silva - UFRGS - Porto Alegre/RS

- Sergio Domingos Pittelli - USP - São Paulo/SP

- Silviamar Camponogara - UFSM - Santa Maria/RS

- Sonia Regina Pasian - USP - Ribeirão Preto/SP

- Taís Mata-Santos - FURG - Rio Grande/RS

- Tânia Maria Valente Pacheco - FIOCRUZ - Rio de Janeiro/RJ

- Thais de Oliveira Gozzo - USP - Ribeirão Preto/SP

- Thiago Simao Gomes - UNIFESP - São Paulo/SP

- Vanessa Emille Carvalho de Sousa - UFC - Fortaleza/CE

- Vanessa Terezinha Gubert de Matos - UFMS - Campo Grande/MS

- Vania Barbosa do Nascimento - FMABC - Santo André/SP 